77 patients (56\%) were readmitted to hospital, 66 (86\%) for clearly alcohol-related reasons. 13 more patients re-attended the AandE Department without readmission. 100 day readmission rate was 50\%. 19 patients were readmitted twice and 23 patients $>3$ times. Readmission was independently associated with unemployment $(\mathrm{p}=0.043)$, self-discharge after index admission $(\mathrm{p}=$ 0.011 ), relapse into drinking $(\mathrm{p}=0.028)$, and (surprisingly) with having received a brief intervention regarding alcohol consumption during the index admission from a dedicated alcohol worker $(\mathrm{n}=61, \mathrm{p}=0.009)$. Seven more patients had died by 21/05/13, 5 from liver disease.

Conclusion Patients admitted to hospital with AUDs tend to be socially deprived, frequent hospital attenders with major physical and mental co-morbidity. They have high subsequent alcohol relapse and hospital readmission rates. Reduction of these is not achieved by interventions during the index admission and will require more pro-active measures post-discharge.

Disclosure of Interest None Declared.

\section{PTH-072 IMPROVING THE QUALITY OF AN ACUTE GI BLEEDING SERVICE: IMPACT OF INTERVENTIONS. RESULTS OF THREE PROSPECTIVE AUDITS IN A TERTIARY CENTRE}

SM Alam*, N Chauhan, K Sager, A Bond, P Collins. Gastroenterology, Royal Liverpool and Broadgreen University Hospital Liverpool UK, Liverpool, UK

\subsection{6/gutjnl-2014-307263.518}

Introduction National guidelines for the management of upper gastrointestinal (GI) bleeding exist and are based on conclusive evidence for effective clinical practice ${ }^{[1]}$. A mortality rate in acute admissions of $7 \%$ was reported in a national audit of upper GI bleeding $^{[2] .}$ This is an area of high volume, high risk and high cost where improvements can be made.

Methods Three prospective audits of all acute admissions with upper GI bleed were undertaken for 4 week periods in 2009 (Audit 1), 2011 (Audit 2) and 2013 (Audit 3). After Audit 1, a new GI bleed proforma was introduced, a rolling,targeted educational programme for Accident and Emergency (AandE) and Medical Admissions Unit (AMAU) trainees was started,mandatory fields for risk scoring were included in the electronic requests and additional evening inpatient endoscopy lists were started. After Audit 2, Saturday and Sunday inpatient endoscopy lists were introduced and a dedicated endoscopy co-ordinator supervised triaging of patients to appropriate lists.

Results A total of 115 patients were included in the three audits. $88 \%$ were admitted through AandE. There were no deaths and no patients underwent surgery in each of the three audit periods. $13 \%$ of all patients had lesions at endoscopy requiring therapy (6\% band ligation for variceal bleeding, 7\% endotherapy for peptic ulcer bleeding). The proportion of patients in whom a risk score was calculated in the 2009, 2011 and 2013 audits improved with each audit period with completion rates of $0 \% .39 \%$ and 94\%, respectively. ( $\mathrm{P}<0.001$ for comparison of 2009 to 2011, and 2011 to 2013). However, the risk scores were inaccurately calculated by the admitting doctors in $46 \%$ and $33 \%$ of cases in Audit 1 and Audit 2. The improvement in accuracy between the audit periods was not statistically significant $(p=0.64)$. There was a statistically significant improvement in the time from admission to endoscopy between the audit periods 2009 and 2013 (median $33.5 \mathrm{~h}$ (range 15 to $214 \mathrm{~h}$ ) versus $23.25 \mathrm{~h}$ (range 1.5 to $92 \mathrm{~h}$ ) ( $\mathrm{p}=$ 0.0017). The proportion of patients having endoscopy within 24 $\mathrm{h}$ of admission improved between audit 1 and Audit 3 (23\% and $46 \%$, respectively $(\mathrm{P}=0.04))$.
Conclusion Targeted interventions have been associated with incremental improvements in the quality of care for patients admitted acutely with acute GI bleeding in the last 4 years. Mortality rates have been consistently well below the national average. Further interventions will include targeted education to improve the accuracy of risk stratification of patients admitted with upper GI blood loss and changes to the mechanism of triage to inpatient endoscopy lists to improve the time from admission to endoscopy.

\section{REFERENCES}

1 NICE(Clinical guideline 141.) 2012

2 Hearnshaw SA, et al. Gut 2010;59:1022-1029

Disclosure of Interest None Declared.

\section{PTH-073 UNITED KINGDOM NATIONAL BOWEL CANCER AWARENESS PROGRAMME - MORE PAIN, NO GAIN?}

${ }^{1} \mathrm{~T}$ Khong*, ${ }^{2} \mathrm{~K}$ Naik, ${ }^{1} \mathrm{R}$ Sivakumar, ${ }^{3} \mathrm{~S}$ Shah. ${ }^{1}$ Deparment of Surgery, Pinderfields Hospital, Mid Yorkshire NHS Trust, Wakefield, UK; ${ }^{2}$ Department of Radiology, Pinderfields Hospital, Mid Yorkshire NHS Trust, Wakefield, UK; ${ }^{3}$ Department of Gastroenterology, Pinderfields Hospital, Mid Yorkshire NHS Trust, Wakefield, UK

\subsection{6/gutjpl-2014-307263.519}

Introduction The UK government embarked on two National Bowel Cancer Awareness Campaigns in 2012 to raise public awareness of colorectal cancer (CRC) and to prompt symptomatic individuals to visit their primary care physicians early. A pilot programme in 2011 failed to demonstrate neither increased numbers, nor earlier stage of new CRC diagnosed, despite significant rise in $2 \mathrm{WW}$ referrals ${ }^{1}$. It is unclear whether such findings would translate to other regions of the UK during a nationwide awareness campaign.

Aims/Objectives

1. To determine the effects of the bowel awareness campaigns on $2 \mathrm{WW}$ referrals.

2. Comparison of the number of CRC cases diagnosed during the campaigns to a comparable period in 2011 .

3. Stage of disease and survival for patients diagnosed during the campaigns.

Methods Retrospective study of over 1439 consecutive patients referred through the $2 \mathrm{WW}$ colorectal pathway to Mid-Yorkshire Hospital NHS Trust during the campaigns between 1/2/2012 to $30 / 4 / 2012$ and $1 / 9 / 2013$ to $31 / 10 / 2012$. Total number of referrals, newly diagnosed cases of CRC and non-CRC, with their respective staging were determined and compared with a comparable group in 2011. One year survival for the two groups was evaluated by Kaplan-Meier.

Results Referrals through the 2WW pathway increased by 55$60 \%$ during the bowel awareness campaigns, but there was no significant relative increase in CRC or non-CRC diagnoses. Positive diagnostic yield for CRC remained low at 5.6\% and $6.1 \%$. The bowel awareness campaigns did not affect the stage at which CRC patients were diagnosed, as over 50\% presented with Stage 3 and 4 disease, and similarly there was overall no difference in 1 year survival.

Conclusion The UK bowel awareness campaign has increased public awareness of CRC and prompted symptomatic individuals to seek medical attention. This study shows the increase in $2 \mathrm{WW}$ referrals has not translated to better outcomes for patients. Furthermore the study highlights the difficulty in assessing a symptomatic individual's risk for CRC in primary care, despite current guidelines which carry poor positive predictive value. 
Better risk assessment tools are desperately required, otherwise, future national campaigns would add significant presures to already overstretched colorectal units.

\section{REFERENCE}

1 Taylor M, COI, Radford G, Network. AC. Evaluation of the Bowel Cancer Awareness Pilot in the South West and East of England 31 January to 18 March 2011. Department of Health UK.; 2012

Disclosure of Interest None Declared.

\section{PTH-074 TRANSFORMING WARD ROUNDS}

V Krishnan*, D Kejariwal. Gastroenterology, University Hospital of North Durham-NHS, Durham, UK

\subsection{6/gutjnl-2014-307263.520}

Introduction Medical 'ward round' (WR) is a complex clinical process and a key component of daily hospital activity. Despite this, there is a clear paucity of quality indicators and evidence base for best practice for WR with considerable variability in the efficiency and quality.

This prompted us to devise and implement a ward round checklist (WRC)based on the Royal College of Physicians (RCP) and Nursing $(\mathrm{RCN})^{[1]}$ to improve quality of inpatient care.

Methods We developed the WRC (Figure 1) for a comprehensive patient review, got approved by the hospital health records committee and used as a sticky note in clinical notes.

The WRC was designed to be used as a memory aid and not to limit critical clinical thinking.

This was piloted in a medical ward and used during every WR.

Results We collected data over a week on ward A (pilot medical ward) and a comparator medical ward (ward X -where WRC was not used).

Among patients in ward A, a subgroup of patients in whom WRC was not used were analysed as a separate sub-group.

Total of 45 patients were assessed during the period, 28 patients from ward A, 19 patients from ward X.

Venous Thrombo-Embolism assessment and action was done in $96.4 \%(27 / 28)$ in ward A (100\% in WRC used WR) whilst it was $73.6 \%(14 / 19)$ in Ward X.

Resuscitation and escalation of care decision was made in $67 \%(19 / 28)$ in ward A (93.3\% in WRC used WR). It was done only in $31.3 \%(6 / 19)$ of patients in Ward X.

Antibiotic stop date was mentioned in $68.7 \%(11 / 16)$ in ward A $(100 \%-7 / 7$ in WRC used WR). It was done in $22.2 \%(2 / 9)$ in Ward X.

\begin{tabular}{ll} 
Abstract PTH-074 Figure 1 & \\
\hline WR by & Reviewed \\
\hline Observation chart & Y N NA \\
Fluid balance and Nutrition & Y N NA \\
VTE risk assessment & Y N NA \\
Drips and catheter needed & Y N NA \\
Antibiotics- appropriate/stop date & Y N NA \\
Drugs chart review & Y N NA \\
Scans and results & Y N NA \\
Resus/ceiling of care/MET call & Y N NA \\
Discharge plan & Y N NA \\
Patient awareness & Y N NA \\
\hline
\end{tabular}

Expected Day of Discharge was mentioned in 65.2\% (15/23 5 patients were very unwell to comment on EDD) in ward A $(76.5 \%-10 / 13$ in WRC used WR). It was done in $53.8 \%$ (7/ 13) of the ward $X$.

Conclusion A recent NEJM article ${ }^{2}$ evaluating the use of checklists for high-fidelity crisis simulation showed an impressive difference in missing critical steps, $6 \%$ with checklists vs $23 \%$ without checklists.

The WHO has already recognised and introduced the surgical safety checklist to reduce morbidity and mortality.

We believe that checklists have the potential to improve patient outcomes by ensuring that all patients receive evidence based best practices and safe high quality care. This allows physicians to concentrate on the higher thinking in WR and WRC to ensure that basics are covered.

\section{REFERENCES}

1 RCP, RCN. Ward rounds in medicine: principles for best practice. London: RCP, 2012

2 Arriaga AF, Bader AM, et al. A simulation-based trial of surgical-crisis checklists. N Engl J Med 2013;368:246-53. DOI: 10.1056/NEJMsa1204720

Disclosure of Interest None Declared.

\section{PTH-075 'ASCITES CLINIC': AN OUTPATIENT SERVICE MODEL FOR PATIENTS REQUIRING LARGE VOLUME PARACENTESIS}

${ }^{1}$ WJ Gashau*, ${ }^{1} \mathrm{G}$ Samra, ${ }^{2} \mathrm{~S}$ Gasser, ${ }^{1} \mathrm{M}$ Rolland, ${ }^{2} \mathrm{P}$ Sambaiah, ${ }^{1} \mathrm{C}$ Shorrock. ${ }^{1}$ Gastroenterology, Blackpool, UK; ${ }^{2}$ Blackpool Teaching Hospitals Foundation Trust, Blackpool, UK

\subsection{6/gutjnl-2014-307263.521}

Introduction A retrospective profile of medical readmissions within 30 days of discharge (September 2011-December 2011) from a busy district teaching hospital highlighted that a small proportion of patients (12\%) with recurrent ascites accounted for $68 \%$ of readmissions. Most required large volume paracentesis (LVP) with a mean length of stay of 4 days. We aimed to determine if a viable, safe model for large volume paracentesis (LVP) in an outpatient setting is feasible.

Methods Changes included identifying motivated liver specialist nurses to lead the ascites clinic service, detailed development of local policy and in-patient referral systems for patients appropriate for the service. Patients are initially reviewed in face-to-face clinics allow comprehensive history, examination and augmentation of information to empower individuals to self-monitor and self-refer based on weight and abdominal girth. These are run in tandem with a consultant led hepatology clinic for senior medical support. Where appropriate, follow up can occur by telephone. If necessary, facilitation of same day elective admission for LVP can be arranged. Competent gastroenterology trainees in the day-case endoscopy unit to carry out LVP with same day patient discharge

Results From September 2012 to May 2013, 68 LVPs have been performed in 12 patients. Complications have been few with only one patient having been admitted twice overnight for ongoing large volume paracentesis. Emergency readmissions for LVP have fallen from $68 \%$ to $13 \%$ over the corresponding period 12 months earlier with an improved patient experience. Conclusion The 'Ascites Pathway' allows safe, effective outpatient LVP with increased patient satisfaction. We feel therefore that LVP is best managed in an outpatient setting with a dedicated nurse-led, medically supported ascites service.

Disclosure of Interest None Declared. 